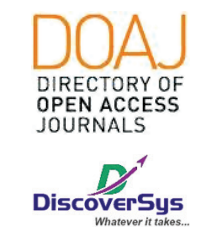

Published by DiscoverSys

\section{Microbiota-based therapies for chronic kidney disease}

\author{
Dessy Natasha Ade Putri, ${ }^{*}$ Chika Christianne Moreen Nababan
}

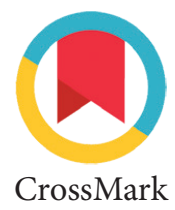

CrossMark

\title{
ABSTRACT
}

Background: Increasing prevalence of chronic kidney disease (CKD) worldwide has been concerned as a global public health issue because of the burden of adverse outcomes, including mortality. Gut microbiota can be used as a potential therapeutic target to slow the progression of CKD. Aim: This literature review aims to provide an in-depth review about the potency of microbiota-based therapy for CKD.

Method: from 58 journals reviewed, 51 were found suitable as references for this paper. The keywords used are "gut microbiota," "chronic kidney disease", "prebiotic", "probiotic" and "fecal microbiota transplantation" on selected search engines.

Result: In general, it has shown that microbiota-based therapies do have potential therapeutic effect as it can improve inflammatory response, uremic toxin, insulin resistance and cardiovascular risk.

Conclusion: Prebiotic, probiotic, fecal microbiota transplantation and novel therapies have shown beneficial effects in treating patients with CKD.
Third Year Medical Student, Medical Faculty of Udayana University, (infofk@unud.ac.id)
${ }^{*}$ Corresponding:

Dessy Natasha Ade Putri, Third Year Medical Student, Medical Faculty of Udayana University dessynatasyaputri@gmail.com

Received: 2018-04-09 Accepted: 2018-06-17 Published: 2019-04-01

Keywords: Gut microbiota, chronic kidney disease, prebiotic, probiotic, fecal microbiota transplantation

Cite This Article: Putri, D.N.A., Nababan, C.C.M. 2019. Microbiota-based therapies for chronic kidney disease. Intisari Sains Medis 10(1): 57-64. D0l: $10.1556 /$ ism.v10i1.239

\section{INTRODUCTION}

Chronic kidney disease (CKD) is a condition in which the kidneys are damaged or cannot filter blood as well as healthy kidneys. Because of this, excess fluid and waste from the blood remain in the body and may cause other health problems. ${ }^{1}$ Chronic kidney disease defined as structural or functional abnormalities of the kidneys for 3 months that manifest by either: Kidney damage (with or without decreased glomerular filtration rate) as defined by pathological abnormalities or markers of kidney damage (albuminuria with AER/albumin excretion rate $\geq 30 \mathrm{mg} / 24$ hours; ACR/albumin creatinine ratio $\geq 30 \mathrm{mg} / \mathrm{g}$ or $3 \mathrm{mg} /$ mmol, urine sediment abnormalities, electrolyte and other abnormalities due to tubular disorders, abnormalities detected by histology, structural abnormalities detected by imaging, history of kidney transplantation), or decrease of GFR below $60 \mathrm{~mL} / \mathrm{min} / 1.73 \mathrm{~m}^{2}$, with or without kidney damage. $^{2}$

Thirty million people or $15 \%$ of United States adults are estimated to have CKD. ${ }^{1}$ The most common cause of chronic, and end-stage renal disease (ESRD) in the US is diabetes which accounts for about $50 \%$ of all cases, followed by hypertension (25\%), with other causes including glomerulonephritis and polycystic kidney disease. Cardiovascular disease remains the leading cause of mortality in patients with CKD. ${ }^{3}$ According to Riset Kesehatan Dasar (RISKESDAS) data in 2013, the prevalence of CKD in Indonesia is $0.2 \%{ }^{4}$
Chronic Kidney Disease is recognized as a global public health issue because of its rising prevalence worldwide (about 10\%) and the burden of adverse outcomes, including mortality that is almost doubled in the last two decades. ${ }^{5}$ Rates of rehospitalization for CKD patients are higher, in 2012, $24 \%$ of patients with CKD were readmitted within 30 days, compared to 17 percent of those without CKD. ${ }^{6}$ Approximately 20 million people in the United States are currently affected by chronic kidney disease (CKD), with half a million of these presenting with the most severe form ESRD. The only treatments for ESRD are dialysis or transplantation. However, the mortality rate of patients on dialysis can be as high as $20 \%$ per year. $^{3}$

Treatment strategies for CKD are targeting potential risk factors for CKD progression such as: hypertension, diabetes mellitus, acute kidney injury, nephrolithiasis, hyperuricemia, metabolic acidosis, socioeconomic factors, metabolic bone disease, renal vasoconstriction and renal fibrosis. ${ }^{7}$ Current treatment strategies focusing on blood pressure control via blockade of the renin-angiotensin-aldosterone (RAAS) system that has a pathophysiologic role in the progression of CKD. ${ }^{3}$ Although ACEIs and ARBs slow the progression of renal disease, they do not halt it and have been associated with side effects including cough (for ACE inhibition) and serious hyperkalemia. ${ }^{3}$ Mechanisms for these adverse effects include preferential constriction of the efferent arteriole leading to glomerular 
hypertension, direct effects of angiotensin II on increasing mesangial cell proliferation and matrix expansion, and promotion of inflammation. ${ }^{8}$

Renal replacement therapy is other option in therapy for CKD. Conventional dialysis techniques, however seem to have reached their limits. This techniques failed to show an improvement of patient outcome by increasing the removal of low molecular mass water soluble solutes and even middle molecules above standard of care. Renal transplantation, undoubtedly the best treatment option in patients with ESRD is hampered by organ shortage, and morbidity related to the surgical procedure and life-long immunosuppression. ${ }^{9}$

Microbiota especially in intestine has been found to be a complex ecosystem with multiple influences on its human host. Understanding the role of human gut microbiota in the progression of CKD requires a clear comprehension of the microbiota composition, dynamics, and stability within a patient. Gut microbes produce compounds that are normally excreted by the kidneys but can be considered as potential uremic retention. ${ }^{10}$

Dysbiosis in CKD patients may contribute to increased uremic toxin levels that in turn contribute to CKD progression..$^{10}$ Modulation of gut microbiota can be as an alternative or adjuvant for therapeutic strategies in a patient with CKD. Gut microbiota based therapy is a potential therapy that targets uremic toxin, inflammation, insulin resistance, and cardiovascular risk in a patient with CKD.

\section{METHODS}

A literature search was performed by PubMed, Scholar, ProQuest search from January 2009 till August 2017 using these following keywords: "gut microbiota", "chronic kidney disease", "prebiotic", "probiotic", "fecal microbiota transplantation". The search strategy conducted by using keyword or combination in the search engine and focus on articles that provided definitive primary data from randomized controlled trials and experimental, but we also included systematic reviews to determine whether these contained any additional information not covered by the primary data. Any study that was deemed potentially relevant for systematic review was retrieved for inspection. Publications of any type were included if they reported original data from such a procedure of microbiota-based therapy for CKD and must have been published in the last 10 years. The publication was excluded if it was published more than 10 years ago and published in a language other than English and Indonesian. From 58 journals reviewed, 51 were found suitable as references for this literature review.

\section{RESULT AND DISCUSSION Gut Microbiota}

The human body consists of up to 100 trillion bacterial cells from more than 500 distinct species, with the gastrointestinal tract being the usual habitat for $>70 \%$ of those microbes. ${ }^{11}$ The human colon is colonized by a diverse and highly abundant microbiota, comprising bacteria, archaea, eukaryotes, and viruses. ${ }^{12}$ In general, the adult gut is dominated by two bacterial phyla, Firmicutes and Bacteroidetes, and with other phyla, including Actinobacteria, Proteobacteria, Verrucomicrobia, Cyanobacteria, Fusobacteria, Spirochaetes, and TM7. ${ }^{13}$

The collective genomic content of the microbiota called microbiome is complex and has been estimated to contain more than 5 million unique genes. Microbiota have a crucial role in the development and homeostasis of the host by building and modulating the immune system and improving the intestinal defense to face opportunistic pathogens. ${ }^{14}$ Under physiologic conditions, it participates in certain complementary metabolic activities that have not been fully evolved in the human host, such as the breakdown of undigestible plant polysaccharides, synthesis of certain vitamins, biotransformation of conjugated bile acids, and degradation of dietary oxalates. ${ }^{13}$ Environmental factors (such as intestinal $\mathrm{pH}$, antibiotics, nutrient intake, psychological and physical stress, intestinal wall edema, iron intake), host genotype, extraintestinal noncommunicable diseases and inflammatory bowel diseases may lead to intestinal dysbiosis. ${ }^{11}$

\section{Microbiome Dysbiosis in CKD}

The presence of CKD/ESRD also contributes significantly to the pathogenesis of intestinal dysbiosis by different mechanisms, linked both to alterations of the intestinal barrier and changes in the composition of gut microbiota and by the effects of dietary restrictions and specific therapies of the syndrome. ${ }^{11}$ Impaired renal function due to diminished glomerular filtration rate and tubular absorption leads to increased levels of compounds that should be eliminated, thus accumulating toxicity. When end products of purine metabolism such as urea, uric acid, and oxalates, reach high levels in the bloodstream, the colon becomes the major excretory organ to maintain body homeostasis. Serum urea accumulation during CKD increases urea influx into the intestinal lumen, where urease-producing bacteria hydrolyze it into ammonia and ammonium hydroxide. It consequently increased intestinal $\mathrm{pH}$ and promoting mucosal irritation and structural alterations to the gut barrier. ${ }^{14}$ Dysfunction of the intestinal barrier in CKD is associated with bacterial translocation and endotoxemia, which is related to systemic 


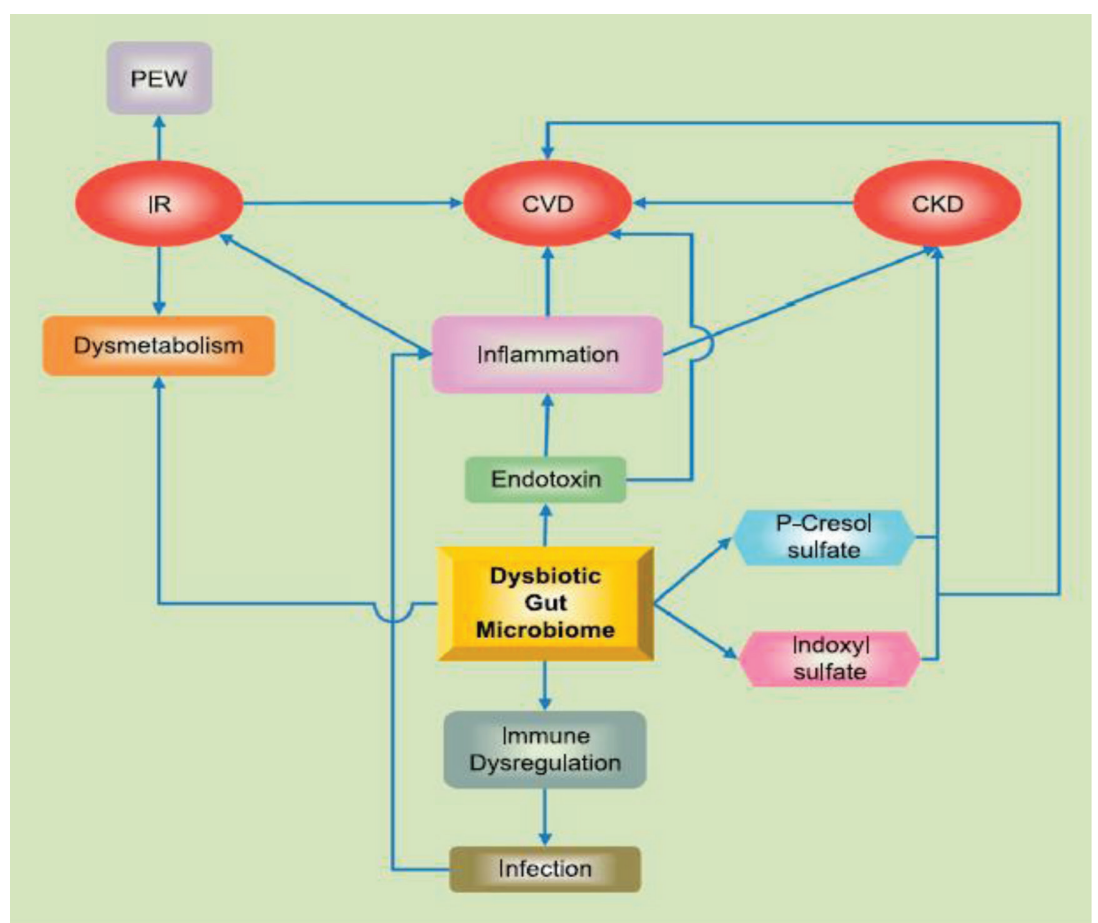

Figure 1 Alterations in gut microbiota and impaired intestinal barrier function in patients with CKD/ESRD have been linked to endotoxemia and accumulation of gut-derived uremic toxins leading to insulin resistance, protein-energy wasting, immune dysregulation, and atherosclerosis. CVD, cardiovascular disease; IR, insulin resistance; PEW, protein-energy wasting ${ }^{13}$

inflammation, malnutrition, cardiovascular disease, and possibly, reduced survival. ${ }^{9}$

Microbial DNA analyses that compare stools from patients with ESRD and healthy persons showed significant differences in the abundance of 190 microbial operational taxonomic units (OTUs) between them. ESRD group is mostly belonged to the Pseudomonadaceae family, whereas healthy individuals mostly belong to the Sutterellaceae, Bacteroidaceae and Lactobacillaceae families. Similar results were obtained with an experimental model of CKD, 5/6 nephrectomy resulted in significant differences in OTU abundance, of which the Bacteroidetes and Firmicutes families, especially Lactobacillaceae and Prevotellaceae, were markedly less prevalent in nephrectomized rats. ${ }^{15}$

\section{Gut Derived Uremic Toxin}

CKD, especially in its most advanced stages, is characterized by the progressive accumulation of many substances and solutes, such as electrolytes, hormones, and uraemic solutes. Uremic solutes are able to interfere with many biological functions and are indicated as uremic toxins. ${ }^{12}$ In the presence of this disordered bacterial colonization, there is increased production of gut-derived uremic toxins such as indoxyl sulfate (IS) and $p$-cresol sulfate. ${ }^{16}$ In healthy subjects, both molecules are excreted by the kidney by active tubular secretion, whereas in CKD, increased blood levels of $p$-cresol sulfate and indoxyl sulfate parallel with renal function reduction. ${ }^{16}$

A study in 24 patients with ESRD exhibited significant expansion of bacterial families possessing urease, uricase, indole sulfate and $p$-cresol forming enzymes and decreased in bacterial families possessing butyrate forming enzymes. Ureasederived ammonia give damaging effects on the intestinal epithelial barrier whereas indoxyl sulfate and $p$-cresol sulfate give toxic effects. Meanwhile, microbial butyrate forming enzymes (Short Chain Fatty Acid - SCFA) give protective effect for the gut barrier. This observed changes in the intestinal microbiota likely contribute to the uremic toxicity and local and systemic inflammation in ESRD population. ${ }^{17}$

In ESRD patients on dialysis, the clearance of $p$-cresol sulphate and indoxyl sulphate is $<10 \%$ compared with normal kidneys, and their blood levels have been related to poor outcomes. ${ }^{12}$ Poesed et al. prospectively followed 488 patients with CKD stages 1-5 showed that serum $p$-cresol sulfate was higher than serum $p$-cresol glucuronide, with a significant correlation between estimated glomerular filtration rate (eGFR) and proportion of serum $\mathrm{p}$-cresol sulfate to glucuronide. Higher serum $\mathrm{p}$-cresol and lower proportion of serum p-cresol sulfate to glucuronide were jointly and significantly associated with mortality and cardiovascular disease. ${ }^{16}$

Serum IS levels were significantly correlated with CKD stages. The total IS and free IS were strongly associated with age, hypertension, cardiovascular disease, blood pressure, pulse wave velocity, blood urea nitrogen, creatine, and phosphate, but negatively correlated with eGFR, hemoglobin, hematocrit, and calcium. ${ }^{18}$ These protein-bound uraemic solutes may in fact negatively affect endothelial function and repair by several mechanisms, including inflammation, oxidative stress, impaired nitric oxide production and inhibition of endothelial proliferation and healing, and they have been associated with increased incidence of CVD and mortality. ${ }^{12}$

Recently, Hazen's group demonstrated that plasma levels of gut microbial-derived trimethylamine-N-oxide (TMAO) correlated with increased 5-year mortality risk in CKD subjects after multivariate adjustment. TMAO, a gut microbial-dependent metabolite of dietary choline, phosphatidylcholine (lecithin) and L-carnitine, is elevated in CKD and associated with coronary artery disease pathogenesis. Dietary TMAO supplementation in animal models resulted in increased tubulointerstitial fibrosis and progressive renal dysfunction. ${ }^{19}$ 


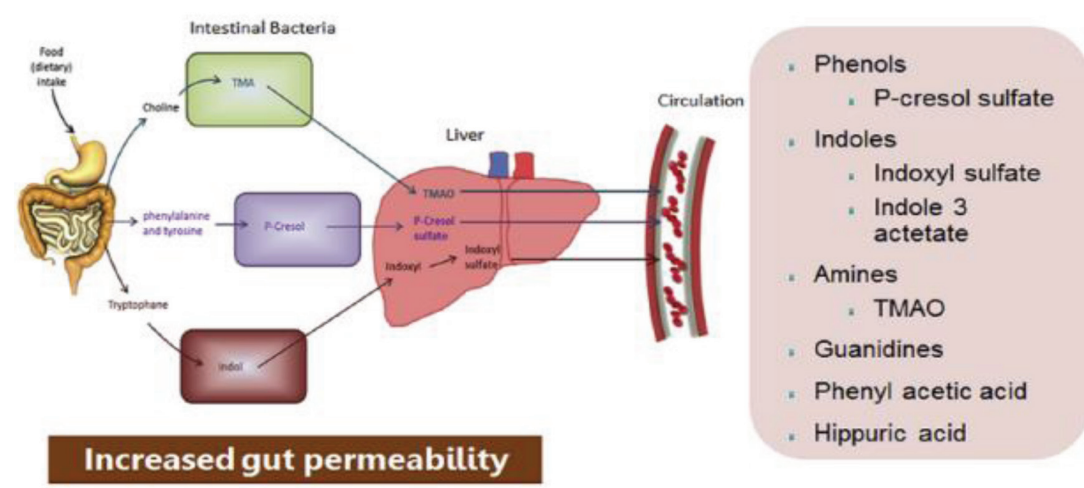

Figure 2 Schematic representation of origin and synthesis of the major uremic toxins from dysbiotic gut microbiome, subsequent modification in the liver, and secretion into the circulation. TMA, trimethylamine;TMAO, tri- methylamine- $\mathrm{N}$-oxide ${ }^{20}$

Tang et al. examined the relationship between fasting plasma TMAO and all-cause mortality over 5-year follow-up in 521 stable subjects with CKD, median TMAO level among CKD subjects was markedly higher than in non-CKD subjects. Among non-CKD subjects, elevated TMAO levels portend poorer prognosis within cohorts of high and low cystatin C. In animal models, elevated dietary choline or TMAO directly led to progressive renal tubulointerstitial fibrosis and dysfunction. ${ }^{21}$

\section{Endotoxin Induce Inflammation in CKD}

Accumulating evidence over the recent years has spotlighted the gastrointestinal tract as a major source of chronic inflammation in CKD. Gut bacterial DNA fragments have been detected in the blood of both pre-dialysis CKD and chronic hemodialysis patients. ${ }^{22}$ In patients who were positive in blood bacteria, the bacterial DNA concentration was positively correlated with plasma levels of C-Reactive Protein and Interleukin 6. The ESRD patients who underwent hemodialysis had a different flora and showed slightly higher levels of high sensitivity CRP, IL-6, and plasma endotoxin, compared with those in ESRD patients who did not undergo hemodialysis. Hemodialysis may exaggerate microinflammation in ESRD patients to some extent. ${ }^{22}$ Shi et al found that bacterial DNAs were detected in the blood of $20 \%(6 / 30)$ of the ESRD patients. All the observed genera in blood (Klebsiella spp, Proteus spp, Escherichia spp, Enterobacter spp, and Pseudomonas spp) were overgrown in the guts of the ESRD patients. Plasma D-lactate, hs-CRP, and IL-6 levels were significantly higher in patients with bacterial DNA than those without. That study concluded that bacterial translocation occurs in ESRD patients and is associated with microinflammation in ESRD ${ }^{23}$

Endotoxin, the hydrophobic anchor of Lipopolysaccharide (LPS), is a phospholipid that constitutes the outer membranes of most Gramnegative bacteria. It is continuously produced in the gut and is transported into intestinal capillaries through a Toll-Like Receptor (TLR-4) dependent mechanism. Endotoxin circulates in the plasma of healthy humans at low concentrations (between 1 and $200 \mathrm{pg} / \mathrm{ml}$ ). Endotoxin stimulates cells of the immune system, particularly macrophages and the endothelial cells, to become activated and to synthesize and secrete a variety of effector molecules that cause an inflammatory response. ${ }^{13}$ Endotoxin is measurable in the blood of dialysis patients and correlates with the severity of systemic inflammation in the absence of clinically detectable infection. That hemodialysis cohort found associations between endotoxinemia and CRP, body composition and High Dense Lipoprotein (HDL). ${ }^{24}$

\section{Insulin Resistance and CKD}

Insulin resistance (IR) refers to reduced sensitivity of organs to insulin-initiated biologic processes that result in metabolic defects. Insulin resistance is common in patients with ESDR but also occurs in patients with CKD, even when the serum creatinine is minimally increased. ${ }^{25}$ The exact mechanisms underlying insulin resistance in patients with CKD have not been elucidated, but growing evidence suggests that the progressive retention of a large number of compounds, which under normal conditions are excreted by the healthy kidneys, could play a key role. ${ }^{26}$

Recent findings suggest that our gut microbiota might be involved in the development of obesity and related disorders, such as insulin resistance. Microbiome dysbiosis can induce uremic toxin and inflammation which trigger insulin resistance. For example, accumulation of $p$-cresol sulfate in patients with CKD or animal models of CKD resulted in impaired insulin-stimulated intracellular signaling. Furthermore, when normal mice were treated with $p$-cresol sulfate, insulin resistance developed and lipids accumulated in muscle and liver. ${ }^{26}$ In cultured 3T3-L1 adipocytes or human adipocytes, the addition of $p$-cresol sulfate has been associated with suppression of lipogenesis and stimulation of lipolysis. Thus, increased levels of the uremic toxin, $p$-cresol sulfate stimulate inflammation and ROS generation leading to changes in insulin-stimulated intracellular signaling and hence, insulin resistance..$^{25}$

\section{Gut Microbiota and Cardiovascular risk}

Gut microbiota-mediated metabolism of phosphatidylcholine, choline, or $L$-carnitine each have been shown to produced trimethylamine- $\mathrm{N}$-oxide $(\mathrm{TMAO}),{ }^{21,27}$ and in multiple clinical studies, TMAO levels have been shown to be associated 
with cardiovascular risks. ${ }^{21,27-29}$ In one large study of subjects with predominantly preserved renal function, Tang et al. reported the prognostic value of TMAO for predicting 3-year risk for major adverse cardiovascular events (myocardial infarction, stroke, or death) remained significant after adjustments for renal function. ${ }^{27}$ Furthermore, animal model studies have revealed that TMAO is mechanistically linked to atherosclerosis development through multiple distinct pathways. Animal model and human clinical studies demonstrate an obligatory role for the gut microbiota in TMAO formation from TMA-containing nutrients, including choline and phosphatidylcholine, $L$ carnitine, and more recently, $\gamma$-butyrobetaine, and to a lesser degree, the choline oxidation metabolite betaine. ${ }^{21}$

Gut bacterial translocation occurs in ESRD patients and is associated with microinflammation in the end-stage renal disease. ESRD patients show a high incidence of microinflammation. This microinflammation contributes to morbidity and mortality in ESRD patients, especially the increased incidence of cardiovascular disease via initiation and aggravation of atherosclerosis. ${ }^{23}$

\section{Modulation of Gut Microbiota Prebiotic}

Prebiotic is a nondigestible (by the host) food ingredient that has a beneficial effect through its selective stimulation of the growth or activity of one or a limited number of bacteria in the colon. ${ }^{13}$ Prebiotics are selective indigestible carbohydrate food sources that promote the growth of Bifidobacteria and Lactobacilli species at the expense of other groups of bacteria in the gut, such as Bacteroides species, Clostridia species, and Enterobacteria. The candidate prebiotics include inulin, fructooligosaccharides, galactooligosaccharides, soya-oligosaccharides, xylo-oligosaccharides, and pyrodextrins. ${ }^{13,30}$

Study about prebiotic amylose maize resistant starch reported that, the high resistant starch diet retards CKD progression. This prebiotic which reaches the colon undigested and is metabolized by bacteria to SCFA, improved creatinine clearance and reduced kidney inflammation and fibrosis. Consumption of high amylose maize resistant starch ameliorated inflammation and oxidative stress, reduced severity of renal injury and dysfunction, as well as increased fecal water content in rats with chronic interstitial nephropathy. ${ }^{31}$

Oral intake of prebiotic oligofructose-enriched inulin ( $\mathrm{p}$-inulin) promotes growth of Bifidobacteria was well tolerated and significantly reduced $p$-cresol generation and $p$-cresol sulfate serum concentrations in haemodialysis patients. ${ }^{32}$ Krishnamurthy et al reported that high dietary total fiber intake is associated with lower risk of inflammation and mortality in kidney disease and these associations are stronger in magnitude in those with kidney disease. ${ }^{33}$ Sirich et al reported that increasing dietary fiber in hemodialysis patients may reduce the plasma levels of the colon-derived solutes indoxyl sulfate and possibly $p$-cresol sulfate without the need to intensify dialysis treatments. ${ }^{34}$ Supplementing the diet of CKD patients with fiber may be a dietary therapy to reduce $p$-cresol and improve stool frequency. ${ }^{35}$

Short-chain fatty acids (SCFAs) are end products produced from the fermentation of complex carbohydrates by the intestinal microbiota, especially by anaerobic bacteria. SCFAs have anti-inflammatory and histone deacetylase-inhibiting properties. Therapy with the three main SCFAs (acetate, propionate, and butyrate) improved renal dysfunction caused by injury. This protection was associated with low levels of local and systemic inflammation, oxidative cellular stress, cell infiltration/activation, and apoptosis. However, it was also associated with an increase in autophagy. ${ }^{30}$

Resveratrol (RSV) is a natural polyphenol that mainly occurs in grapes, berries, and other dietary constituents. A study in vivo found that RSV consumption can significantly modulate the growth of specific gut microbiota, including increasing the Bacteroidetes-to-Firmicutes ratios, and the growth of Bacteroides, Lactobacillus, and Bifidobacterium. These results suggested that RSV could be a good candidate as prebiotics and could be used to promote the growth of beneficial commensals and thus to confer health benefits to the host. Meanwhile, RSV decreased TMAO levels by inhibiting commensal microbial trimethylamine (TMA) production via gut microbiota remodeling in mice. ${ }^{36}$

\section{Probiotic}

Probiotics are natural or genetically modified microorganisms expressing specific exogenous enzymes that are able to survive stomach acid and bile, to increase the colon concentration of symbiotons, and confer a health benefit. ${ }^{37}$ The use of probiotics has beneficial effects on the blood pressure, lipid and glycemic profiles, and blood levels of inflammatory biomarkers, as well as the markers of kidney function. This can, therefore, slow down the progression of CKD and reduce cardiovascular complications as the main cause of death in these patients. The main effect of probiotics is characterized by stabilization and adjustment of the intestinal flora. The study showed that taking a synbiotic (synergistic combinations of probiotics and prebiotics) supplement, $500 \mathrm{mg}$, twice a day for 6 weeks, could reduce blood urea nitrogen in patients with 
CKD at stages 3 and $4 .{ }^{38}$ Guida et al examined thirty CKD patients at stages 3 and 4 that were randomized to receive either synbiotic or placebo for four weeks reported that, there was lowered total plasma $p$-cresol concentrations in non-dialyzed CKD patients. High plasma concentrations of $p$-cresol in early phases of $\mathrm{CKD}$ are predictive of progression to end-stage renal disease. ${ }^{39}$

Fang et al in vitro study reported that oral administration of lactic acid bacteria (LAB) Pm1-strain probiotic in cisplatin-induced acute kidney injury model significantly suppressed the accumulation of IS (indoxyl sulphate) in the serum. This finding suggest that treatment with the strain combination Pm1 might be a useful approach to decrease IS accumulation in the serum and kidney. ${ }^{40}$ Study by Akoglu et al using Lactobacillus casei Shirota (LcS) probiotic with 142 patients reported that in patients with initial diagnosed acute renal failure there was a significant improvement of the glomerular filtration rate in the $L c S$ treatment group and the creatinine values dropped significantly on day six and seven in the LcS treatment group with acute renal failure. ${ }^{41}$ Probiotic consumption was associated with a significant increase in natural killer (NK) cell compared with placebo and tended to produce. Additionally, there was a trend towards an increased ratio of IL-10 to IL-12 in association with $L c S$ consumption which indicates a shift toward a more anti-inflammatory profile. ${ }^{42,43}$

Alatriste et al in simple randomized, controlled clinical trial reported that in patients with CKD stage 3 and stage 4, there is a greater than $10 \%$ decrease in the serum urea concentrations after a conventional dietetic treatment with Lactobacillus casei Shirota ( $L c S) . L c S$ dose of $6 \times 10^{9} \mathrm{CFU}$ resulted in a greater decrease of the blood urea level. Probiotics help intestinal microbiome decrease the bacteria producing uremic toxins. ${ }^{44}$ Recent data indicate that renal inflammation is associated with the production of uremic toxins by the intestine and with augments intestinal permeability, leading to the onset of systemic inflammation. ${ }^{30}$

\section{Fecal Microbiota Transplantation (FMT)}

Fecal microbiota transplantation (FMT) involves procedures to the administration of fecal material containing distal gut microbiota from a healthy donor to a patient with a disease or condition related to dysbiosis gut microbiota. The goal of FMT is to treat disease by restoring phylogenetic diversity and microbiota more typical of a healthy individual. ${ }^{45}$ Healthy donors are usually recruited from family members or another healthy person. Potential donors should be questioned about their travel history, sexual behavior, previous operations, blood transfusions, and other factors that increase the risk for the transmissible disease. Potential donors are also screened for family history of autoimmune and metabolic diseases, as well as malignancies (in the first and second degree of family members). Once a donor is selected, blood and fecal samples must be screened for pathogens. ${ }^{46}$

FMT is becoming increasingly accepted as an effective and safe intervention in patients with recurrent Clostridium difficile infection for the restoration of a disrupted microbiome. A case showed successful eradication of a pathogenic organism in a patient with ESRD. ${ }^{10}$ In CKD patient the most abundant microbial families are microbes that able to produce indole and $p$-cresol such as Clostridiaceae, Enterobacteriaceae, and Verrucomicrobiacea, whereas microbial families with butyrate-producing enzymes (Lactobacillaceae and Prevotellaceae) were reduced compared with healthy subjects. ${ }^{17}$ FMT in 16 patient who is receiving active treatment subjectively felt better at 7 weeks and were, therefore, offered weekly FMT enemas for six additional weeks. Interestingly, bacterial diversity was not affected by FMT, but butyrate-producing bacteria including Ruminococcus, Blautia, and Lachnospiraceae were increased in feces from patients that responded to FMT. This change is important since butyrate is a short chain fatty acid that enhances intestinal epithelial cell integrity and anti-inflammatory immune responses. ${ }^{47}$

\section{Novel Therapies}

Lubiprostone is a synthetic derivative of prostaglandin, which activates chloride channel in the gut and is used in the treatment of constipation. ${ }^{20}$ Lubiprostone is a novel laxative that promotes defecation by activating $\mathrm{ClC}-2$ on the apical membrane of the small intestinal epithelium, thereby increasing water secretion into the intestinal lumen to soften feces and enhance intestinal transit. ${ }^{48}$ Oral administration of lubiprostone $(500 \mathrm{mg} / \mathrm{kg}$ per day) changed the fecal and intestinal properties in mice with renal failure. Treatment with lubiprostone reduced the elevated BUN and protected against tubulointerstitial damage, renal fibrosis, and inflammation. Gut microbiome analysis of $16 \mathrm{~S}$ rRNA showed improvement in microbiome profile especially the recovery of the levels of the Lactobacillaceae family and Prevotella genus, which were significantly reduced in the renal failure mice. They also noted that microbiome-derived uremic toxins such as IS and TMAO decreased, and the citric acid cycle pathway was activated indicating improved energy metabolism. ${ }^{49}$ A study in 28 patients who undergo hemodialysis also found that lubiprostone not only improved bowel control but also decreased serum inorganic phosphorus levels that enhance prognosis CKD patients. ${ }^{48}$ 
Trimethylamine (TMA) inhibitor: 3, 3-dimethyl1- butanol (DMB) is a structural analog of choline which inhibits microbial TMA formation through inhibition of microbial TMA lyases. ${ }^{50}$ DMB decreases plasma TMAO levels in mice placed chronically on choline or carnitine-supplemented diet. Furthermore, DMB inhibited endogenous macrophage foam cell formation and atherosclerotic lesion development in mice. DMB was detected in some balsamic vinegar, red wines, and in some olive oils and grape seed oils. ${ }^{20} \mathrm{DMB}$ treatment had significantly reduced plasma TMAO levels and prevented cardiac dysfunction in mice fed with western diet. ${ }^{51}$

\section{CONCLUSION}

The current studies about the role of gut microbiota in the progression of CKD have led to a promising strategy for a more comprehensive therapeutic approach. Microbiota has the potential therapeutic effect that targets uremic toxin, inflammation, insulin resistance, and cardiovascular risk in a patient with CKD. Modulation of gut microbiota was also associated with decreasing of serum urea concentration, blood urea level, serum inorganic phosphorus level, improvement of GFR, creatinine clearance and reduced kidney fibrosis. This modulation can be done by giving prebiotic, probiotic, fecal microbiota transplantation, and novel therapies. More basic and clinical research are needed for further understanding about the role of gut microbiota to slow the progression of CKD and reduce its complications.

\section{ACKNOWLEDGMENT}

The authors would like to thank Medical Faculty of Udayana University for support us.

\section{CONFLICT OF INTEREST}

The authors have no other relevant affiliations or financial involvement with any organization or entity with a financial interest in or financial conflict with the subject matter or materials discussed in the manuscript apart from those disclosed.

\section{REFERENCE}

1. Stats F. National Chronic Kidney Disease Fact Sheet, 2017 CKD Is Common Among Adults in the United States by controlling your blood. 2017;1-4.

2. Ckd DOF, Graded N. Chapter 1: Definition and classification of CKD. Kidney Int Suppl [Internet]. 2013;3(1):19-62. Available from: http://linkinghub.elsevier.com/retrieve/ pii/S2157171615311011
3. Breyer MD, Susztak K. The next generation of therapeutics for chronic kidney disease. Nat Rev Drug Discov [Internet]. 2016;15(8):568-88. Available from: http://dx. doi.org/10.1038/nrd.2016.67

4. Penelitian B, Pengembangan DAN. RISET KESEHATAN DASAR. 2013;

5. Minutolo R, Lapi F, Chiodini P, Simonetti M, Bianchini E, Pecchioli S, et al. Article Risk of ESRD and Death in Patients with CKD Not Referred to a Nephrologist : A 7-Year Prospective Study. 2014;9.

6. Kidney N, Kidney F, Outcomes D, Initiative Q, Kdoqi NKF, Report AD, et al. CKD in the United States: An Overview of the USRDS Annual Data Report, Volume 1. 2015;1:1-10.

7. Campbell D, Weir MR. Defining, Treating, and Understanding Chronic Kidney Disease - A Complex Disorder. 2015;17(7).

8. Drawz PE, Rosenberg ME. Slowing progression of chronic kidney disease. 2013;3(4):372-6.

9. Poesen R, Meijers B, Evenepoel P. The Colon: An Overlooked Site for Therapeutics in Dialysis Patients. Semin Dial. 2013;26(3):323-32.

10. Al Khodor S, Shatat IF. Gut microbiome and kidney disease: a bidirectional relationship. Pediatr Nephrol. 2017;32(6):921-31.

11. Walker AW, Duncan SH, Louis P, Flint HJ. Phylogeny, culturing, and metagenomics of the human gut microbiota. Trends Microbiol [Internet]. 2014;22(5):267-74. Available from: http://dx.doi.org/10.1016/j.tim.2014.03.001

12. Sabatino A, Regolisti G, Brusasco I, Cabassi A, Morabito S, Fiaccadori E. Alterations of intestinal barrier and microbiota in chronic kidney disease. Nephrol Dial Transplant. 2015;30(6):924-33.

13. Ramezani A, Raj DS. The gut microbiome, kidney disease, and targeted interventions. J Am Soc Nephrol [Internet]. 2014;25(4):657-70. Available from: http://www.ncbi.nlm. nih.gov/pubmed/24231662

14. Felizardo RJF, Castoldi A, Andrade-Oliveira V, Camara NOS. The microbiota and chronic kidney diseases: a double-edged sword. Clin Transl Immunol. 2016;5(6):e86.

15. Vaziri ND, Wong J, Pahl M, Piceno YM, Yuan J, Desantis TZ, et al. Chronic kidney disease alters intestinal microbial flora. Kidney Int [Internet]. 2013;83(2):308-15. Available from: http://dx.doi.org/10.1038/ki.2012.345

16. Poesen R, Evenepoel P, de Loor H, Kuypers D, Augustijns P, Meijers B. Metabolism, Protein Binding, and Renal Clearance of Microbiota-Derived $p$-Cresol in Patients with CKD. Clin J Am Soc Nephrol [Internet]. 2016;11:1-9. Available from: http://cjasn.asnjournals.org/ cgi/doi/10.2215/CJN.00160116

17. Wong J, Piceno YM, DeSantis TZ, Pahl M, Andersen GL, Vaziri ND. Expansion of urease- and uricase-containing, indole- and $p$-cresol-forming and contraction of shortchain fatty acid-producing intestinal microbiota in ESRD. Am J Nephrol. 2014;39(3):230-7.

18. Lin C-J, Wu C-J, Wu P-C, Pan C-F, Wang T-J, Sun F-J, et al. Indoxyl Sulfate Impairs Endothelial Progenitor Cells and Might Contribute to Vascular Dysfunction in Patients with Chronic Kidney Disease. Kidney Blood Press Res. 2016;41(6):1025-36.

19. Lau WL, Kalantar-Zadeh K, Vaziri ND. The Gut as a Source of Inflammation in Chronic Kidney Disease. Nephron. 2015;130(2):92-8.

20. Nallu A, Sharma S, Ramezani A, Muralidharan J, Raj D. Gut microbiome in chronic kidney disease: challenges and opportunities. Transl Res [Internet]. 2017;179:24-37. Available from:http://dx.doi.org/10.1016/j.trsl.2016.04.007

21. Tang WHW, Wang Z, Kennedy DJ, Wu Y, Buffa JA, Agatisa-Boyle B, et al. Gut microbiota-dependent trimethylamine N-oxide (TMAO) pathway contributes to both developments of renal insufficiency and mortality risk in chronic kidney disease. Circ Res. 2015;116(3):448-55. 
22. Wang F, Zhang P, Jiang H, Cheng S. Gut bacterial translocation contributes to microinflammation in experimental uremia. Dig Dis Sci. 2012;57(11):2856-62.

23. Wang F, Jiang H, Shi K, Ren Y, Zhang P, Cheng S. Gut bacterial translocation is associated with microinflammation in end-stage renal disease patients. Nephrology. 2012;17(8):733-8.

24. Daniell H. NIH Public Access. Examining Assoc Circ Endotoxin with Nutr Status, Inflamm Mortal Hemodial Patients. 2012;76(October 2009):211-20.

25. Perry RJ, Samuel VT, Petersen KF, Shulman GI, Haven N, Haven N. HHS Public Access. Mol Mech Insul Resist Chronic Kidney Dis. 2015;510(7503):84-91.

26. Koppe L, Pillon NJ, Vella RE, Croze ML, Pelletier CC, Chambert $\mathrm{S}$, et al. $p$-Cresyl sulfate promotes insulin resistance associated with CKD. J Am Soc Nephrol [Internet]. 2013;24(1):88-99. Available from: http://www.ncbi.nlm. nih.gov/pubmed/23274953\%5Cnhttp://jasn.asnjournals. org/content/24/1/88.full.pdf

27. Tang WHW, Wang Z, Levison BS, Koeth RA, Britt $\mathrm{EB}, \mathrm{Fu} \mathrm{X}$, et al. Intestinal Microbial Metabolism of Phosphatidylcholine and Cardiovascular Risk. N Engl J Med [Internet]. 2013;368(17):1575-84. Available from: http://www.nejm.org/doi/abs/10.1056/NEJMoa1109400

28. Moraes C, Fouque D, Amaral ACF, Mafra D. Trimethylamine N-Oxide From Gut Microbiota in Chronic Kidney Disease Patients: Focus on Diet. J Ren Nutr [Internet]. 2015;25(6):459-65. Available from: http:// dx.doi.org/10.1053/j.jrn.2015.06.004

29. Xu K-Y, Xia G-H, Lu J-Q, Chen M-X, Zhen X, Wang $S$, et al. Impaired renal function and dysbiosis of gut microbiota contribute to increased trimethylamine$\mathrm{N}$-oxide in chronic kidney disease patients. Sci Rep [Internet]. 2017;7(1):1445. Available from: http://www.ncbi.nlm.nih.gov/pubmed/28469156\% 5Cnhttp://www.pubmedcentral.nih.gov/articlerender. fcgi?artid=PMC5431124\%5Cnhttp://www.nature.com/ articles/s41598-017-01387-y

30. Andrade-Oliveira V, Amano MT, Correa-Costa M, Castoldi A, Felizardo RJF, de Almeida DC, et al. Gut Bacteria Products Prevent AKI Induced by Ischemia-Reperfusion. J Am Soc Nephrol [Internet]. 2015;26(8):1877-88. Available from: http://www.ncbi.nlm.nih.gov/pubmed/25589612

31. Vaziri ND, Liu SM, Lau WL, Khazaeli M, Nazertehrani S, Farzaneh SH, et al. High amylose resistant starch diet ameliorates oxidative stress, inflammation, and progression of chronic kidney disease. PLoS One. 2014;9(12):1-15.

32. Meijers BKI, De Preter V, Verbeke K, Vanrenterghem Y, Evenepoel P. P-Cresyl sulfate serum concentrations in haemodialysis patients are reduced by the prebiotic oligofructose-enriched inulin. Nephrol Dial Transplant. 2010;25(1):219-24.

33. Krishnamurthy VMR, Wei G, Baird BC, Murtaugh M, Chonchol MB, Raphael KL, et al. High dietary fiber intake is associated with decreased inflammation and all-cause mortality in patients with chronic kidney disease. 2011;81(3):300-6.

34. Sirich TL, Plummer NS, Gardner CD, Hostetter TH, Meyer TW. Effect of increasing dietary fiber on plasma levels of colon-derived solutes in hemodialysis patients. Clin J Am Soc Nephrol. 2014;9(9):1603-10.

35. Salmean YA, Segal MS, Palii SP, Dahl WJ. Fiber supplementation lowers plasma $p$-cresol in chronic kidney disease patients. J Ren Nutr [Internet]. 2015;25(3):316-20. Available from: http://dx.doi.org/10.1053/j.jrn.2014.09.002

36. Chen ML, Yi L, Zhang Y, Zhou X, Ran L, Yang J, et al. Resveratrol attenuates trimethylamine-N-oxide (TMAO)induced atherosclerosis by regulating TMAO synthesis and bile acid metabolism via remodeling of the gut microbiota. MBio. 2016;7(2):1-14.

37. Koppe L, Mafra D, Fouque D. Probiotics and chronic kidney disease. Kidney Int [Internet]. 2015;88(5):1-9. Available from: http://www.nature.com/doifinder/10.1038/ki.2015.255

38. Dehghani H, Heidari F, Mozaffari-khosravi H, Nourimajelan N, Dehghani A. Synbiotic Supplementations for Azotemia in Patients With Chronic Kidney Disease A Randomized Controlled Trial. 2016;
39. Guida B, Germanò R, Trio R, Russo D, Memoli B, Grumetto L, et al. Effect of short-term synbiotic treatment on plasma $p$-cresol levels in patients with chronic renal failure: a randomized clinical trial. Nutr Metab Cardiovasc Dis. 2014;

40. Fang C, Lu J, Chen B, Wu C, Chen Y, Chen M. Selection of uremic toxin-reducing probiotics in vitro and in vivo. J Funct Foods. 2014;7(50):407-15.

41. Akoglu B, Loytved A, Nuiding H, Zeuzem S, Faust D. Probiotic Lactobacillus casei Shirota improves kidney function, inflammation and bowel movements in hospitalized patients with acute gastroenteritis - A prospective study. J Funct Foods [Internet]. 2015;17(August 2015):305-13. Available from: http://linkinghub.elsevier. com/retrieve/pii/S1756464615002595

42. Dong H, Rowland I, Thomas L V., Yaqoob P. Immunomodulatory effects of a probiotic drink containing Lactobacillus casei Shirota in healthy older volunteers. Eur J Nutr. 2013;52(8):1853-63.

43. Reale M, Boscolo P, Bellante V, Tarantelli C, Di Nicola M, Forcella L, et al. Daily intake of Lactobacillus casei Shirota increases natural killer cell activity in smokers. Br J Nutr. 2012;108:308-14.

44. Miranda Alatriste PV, Urbina Arronte R, Gómez Espinosa CO, Espinosa Cuevas MDLÁ. Effect of probiotics on human blood urea levels in patients with chronic renal failure. Nutr Hosp [Internet]. 2014;29(3):582-90. Available from: http://search.ebscohost.com/login.aspx? direct $=$ true $\&$ profile $=$ ehost $\&$ scope $=$ site $\&$ authtype $=$ crawler $\&$ jrnl=02121611\&AN=94645722\&h=OfTXhik DxAZmXS6TEBYMwbGynuJ198FPZV3VphgY5acnJ AB FoK15zrvxQmNI0IncOAGfwO08/QofP30QJXSikw==\& $\mathrm{crl}=\mathrm{c} \% 5 \mathrm{Cnhttp}: / /$ www.ncbi.nlm.nih.gov/pubmed/2

45. Kelly CR, Kahn S, Kashyap P, Laine L, Rubin D, Atreja A, et al. Update on Fecal Microbiota Transplantation 2015: Indications, Methodologies, Mechanisms, and Outlook. Gastroenterology [Internet].2015;149(1):223-37. Available from: http://dx.doi.org/10.1053/j.gastro.2015.05.008

46. Smits LP, Bouter KEC, De Vos WM, Borody TJ, Nieuwdorp M. Therapeutic potential of fecal microbiota transplantation. Gastroenterology [Internet]. 2013;145(5):946-53. Available from: http://dx.doi. org/10.1053/j.gastro.2013.08.058

47. Hansen JJ, Sartor RB. Therapeutic Manipulation of the Microbiome in IBD: Current Results and Future Approaches HHS Public Access. Curr Treat Options Gastroenterol. 2015;13(1):105-20.

48. Gen S, Nobe K, Ikeda N. Lubiprostone, a novel laxative, might improve hyperphosphatemia without water dilution. Ren Replace Ther [Internet]. 2016;2(1):50. Available from: http://dx.doi.org/10.1186/s41100-016-0064-9

49. Mishima E, Fukuda S, Shima H, Hirayama A, Akiyama Y, Takeuchi Y, et al. Alteration of the Intestinal Environment by Lubiprostone Is Associated with Amelioration of Adenine-Induced CKD. J Am Soc Nephrol [Internet]. 2015;26(8):1787-94. Available from: http://www.ncbi.nlm. nih.gov/pubmed/25525179

50. Papageorgiou N, Zacharia E, Briasoulis A, Charakida M, Tousoulis D. Celecoxib for the treatment of atherosclerosis. Non-lethal Inhib gut Microb trimethylamine Prod Treat Atheroscler [Internet]. 2016;25(5):619-33. Available from: http://www.tandfonline.com/doi/full/10.1517/1354 3784.2016.1161756

51. Chen $\mathrm{K}$, Zheng $\mathrm{X}$, Feng $\mathrm{M}$, Li D, Zhang H. Gut microbiota-dependent metabolite Trimethylamine $\mathrm{N}$-oxide contributes to cardiac dysfunction in western diet-induced obese mice. Front Physiol. 2017;8(MAR):1-9.

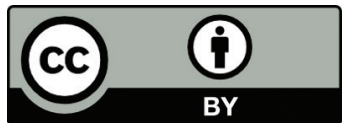

This work is licensed under a Creative Commons Attribution 